\title{
A CFD Study of the Effects of Cone Dimensions on the Flow Field of Cyclone Separators using LES
}

\author{
Kh. Elsayed* and C. Lacor**
}

\begin{abstract}
The effects of the cone dimensions on the flow field in a cyclone separator were investigated computationally. Three cyclones with different cone tip diameters were studied using the commercial CFD code FLUENT. The difficulty of modeling high swirling flow is overcome by means of Reynolds Stress Model (RSM) and Large Eddy Simulation (LES). The axial and tangential velocities in the cyclone are successfully simulated and analyzed with the aid of velocity components, static pressure and dynamic pressure contour plots for the flow field. Results obtained demonstrate that LES is a suitable approach for modeling the effects of cyclone dimensions on the flow field.
\end{abstract}

Keywords: Cyclone Separator, Cone Dimensions, Large Eddy Simulation.

\section{Introduction}

Gas cyclone separators are widely used in industries to separate dust from gas or for product recovery because of their geometrical simplicity and relative economy in power consumption. Cyclones may also be adapted for use in extreme operating conditions (high temperature, high pressure, and corrosive gases). Since there are no moving parts, cyclones are relatively maintenance-free. Therefore, cyclones have found increasing utility in the field of air pollution, the petrochemical and process industries to separate dust from gas streams or for product recovery. Until now, a considerable number of experimental investigations has been performed either on small sampling cyclones or on larger industrial cyclone separators, for example; [1-4]. In these studies, almost all of the eight cyclone dimensions appearing in table 1 , were varied and the changes in cyclone performance characteristics brought about by these variations were studied. However, very little information is available on the effects of the cone bottom (tip) diameter, which determines the cone shape if other cyclone dimensions are fixed [5]. Regarding the effects of change in this dimension, discrepancies and uncertainties exist in the literature. Bryant et al. [6] observed that if the vortex touched the cone wall, particle re-entrainment occurred and efficiency decreased, so collection efficiency will be lower for cyclones with a small cone opening (cone tip diameter). While according to other researchers [5], a cone is not essential for cyclone operation, although it serves the practical purpose of delivering collected particles to the central discharge point. However, Zhu and Lee [7] stated that the cone provides greater tangential velocities near the bottom for removing smaller particles.

\footnotetext{
* PhD researcher, Department of Mechanical Engineering, Vrije Universiteit Brussel, Pleinlaan 2-1050 Brussels- Belgium, /Email khairy.elsayed@vub.ac.be.

** Professor, Department of Mechanical Engineering, Vrije Universiteit Brussel, Pleinlaan 2 1050 Brussels- Belgium, /Email khairy.elsayed@vub.ac.be.
} 
Although the understanding and knowledge of the flow field inside a cyclone has been developed rapidly over the last few years, the exact mechanisms of removing particles are still not fully understood. Therefore, most existing cyclone theories are based on simplified models or depend upon empirical correlations [8]. Xiang et al. [5] carried out experiments with cyclones of different cone dimensions. They evaluated a few models, namely Barth (1956) [9], Leith and Licht (1972) [10], and Iozia and Leith (1990) [11]. All these models were able to simulate correctly the trend of Xiang's experimental data. However, the quantitative agreement was not satisfactory. CFD has a great potential to predict the flow field characteristics and particle trajectories as well as the pressure drop inside the cyclone [12]. Chuah et al. [8] carried out a numerical investigation on the same cyclone dimensions used by Xiang et al. [5] with the commercial finite volume code FLUENT. Using different turbulence models they proved that FLUENT with Reynolds Stress Turbulence Model (RSTM) predicts well the cyclone collection efficiency and pressure drop.

The CFD simulation results from Chuah et al. [8] agree well with Xiang's experimental results in that cyclones with a smaller cone diameter result in a slightly higher collection efficiency compared to cyclones with a bigger cone tip diameter (only if the cone tip diameter is not smaller than the gas exit tube diameter). Also the change in pressure drop will not be significant when the cone size is varied. Both Xiang and Chuah did not give any results about the effect of the cone tip diameter on the flow field inside cyclone separator, except some plots for axial and tangential velocity profiles at two stations in the flow field presented by Chuah et al. [8].

Currently a better understanding of the flow field inside cyclone separators is an important concern, especially with the application of Large Eddy Simulation (LES). The present study was undertaken in an effort to carry out a numerical study on the effect of the cone tip diameter on the flow field and the cyclone performance using FLUENT 6.3 with LES.

\section{Cyclone Design}

The current study deals with the reverse flow cyclone with a tangential rectangular inlet as shown in Fig. 1, the geometrical dimensions of the three cyclones are given in table 1, these dimensions are the same used by Xiang et al. [5] and Chuah et al. [8].

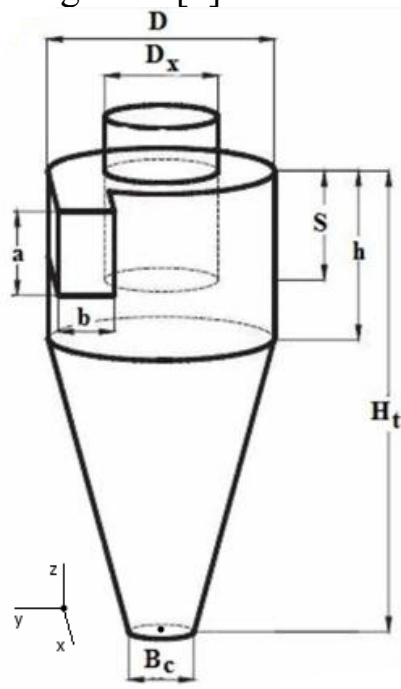

Fig. 1: The cyclone geometry 
Table 1: Geometrical Dimensions of Cyclones Studied

\begin{tabular}{|ll|c|c|}
\hline \hline Dimension & $\begin{array}{c}\text { Length } \\
(\mathrm{mm})\end{array}$ & $\begin{array}{c}\text { Dimension ratio } \\
\text { (dimension/D) }\end{array}$ \\
\hline Body diameter, $D$ & 31 & 1 \\
Gas outlet diameter, $D_{x}$ & & 15.5 & 0.5 \\
Inlet height, $a$ & 12.5 & 0.4 \\
Inlet width, $b$ & 5 & 0.16 \\
Cyclone height, $H_{t}$ & & 77 & 2.5 \\
Cylinder height, $h$ & & 31 & 1 \\
Gas outlet duct length, $S$ & & 15.5 & 0.5 \\
& & & \\
Cone tip diameter, $B_{c}$ & Cyclone I & 19.4 & 0.625 \\
& Cyclone II & 15.5 & 0.5 \\
\hline \hline
\end{tabular}

\section{Computational Fluid Dynamics Simulation}

FLUENT is a commercially available CFD code which utilizes the finite volume formulation to carry out coupled or segregated calculations (with reference to the conservation of mass, momentum and energy equations). For the turbulent flow in cyclones, the key to the success of CFD lies with the accurate description of the turbulent behavior of the flow [12]. To model the swirling turbulent flow in a cyclone separator, there are a number of turbulence models available in FLUENT. These range from the standard $k-\varepsilon$ model to the more complicated Reynolds stress model (RSM) and Large Eddy Simulation (LES). The $k-\varepsilon$ model involves the solution of transport equations for the kinetic energy of turbulence and its dissipation rate and the calculation of a turbulent contribution to the viscosity at each computational cell. The standard $k-\varepsilon$, RNG $k-\varepsilon$ and Realizable $k-\varepsilon$ models were not optimized for strongly swirling flows found in cyclones [8]. The Reynolds stress model requires the solution of transport equations for each of the Reynolds stress components as well as for dissipation transport without the necessity to calculate an isotropic turbulent viscosity field. The Reynolds stress turbulence model yields an accurate prediction on swirl flow pattern, axial velocity, tangential velocity and pressure drop on cyclone simulation [13].

\subsection{Model Description}

\subsubsection{The Reynolds stress transport equations}

RSM foregoes the assumption of isotropic turbulence assumed by $k-\varepsilon$ model and its alternatives and solves a transport equation for each component of the Reynolds stress. It is regarded as the most applicable turbulent model for cyclone flow even though it has the disadvantage of being computationally more expensive in comparison with the other RANS models [14].

\subsubsection{The large eddy simulation (LES) model}

Turbulent flows are characterized by eddies with a wide range of length and time scales. The largest eddies are typically comparable in size to the characteristic length of the mean flow. The smallest scales are responsible for the dissipation of turbulence kinetic energy. It is possible, in theory, to directly resolve the whole spectrum of turbulent scales using an 
approach known as direct numerical simulation (DNS). No modeling is required in DNS. However, DNS is not feasible for practical engineering problems involving high Reynolds number flows. In LES, large eddies are resolved directly, while small eddies are modeled. Large eddy simulation (LES) thus falls between DNS and RANS in terms of the fraction of the resolved scales. The rationale behind LES can be summarized as follows: Momentum, mass, energy, and other passive scalars are transported mostly by large eddies. Large eddies are more problem-dependent. They are dictated by the geometries and boundary conditions of the flow involved. Small eddies are less dependent on the geometry, tend to be more isotropic, and are consequently more universal. The chance of finding a universal turbulence model is much higher for small eddies.

Resolving only the large eddies allows one to use a much coarser mesh and larger times-step sizes in LES than in DNS. However, LES still requires substantially finer meshes than those typically used for RANS calculations. In addition, LES has to be run for a sufficiently long flow-time to obtain stable statistics of the flow being modeled. As a result, the computational cost involved with LES is normally orders of magnitudes higher than that for steady RANS calculations in terms of memory (RAM) and CPU time. Therefore, high-performance computing (e.g., parallel computing) is a necessity for LES, especially for industrial applications, which is the case for the cyclone separator.

\section{Filtered Navier-Stokes Equations}

The governing equations employed for LES are obtained by filtering the time-dependent Navier-Stokes equations in either Fourier (wave-number) space or configuration (physical) space. The filtering process effectively filters out the eddies whose scales are smaller than the filter width or grid spacing used in the computations. The resulting equations thus govern the dynamics of large eddies.

A filtered variable (denoted by an overbar) is defined by

$$
\bar{\phi}(x)=\int_{D} \phi\left(x^{\prime}\right) G\left(x, x^{\prime}\right) d x^{\prime}
$$

where $D$ is the fluid domain and $G$ is the filter function that determines the scale of the resolved eddies. In FLUENT, the finite-volume discretization itself implicitly provides the filtering operation:

$$
\bar{\phi}(x)=\frac{1}{V} \int_{V} \phi\left(x^{\prime}\right) d x^{\prime}, x^{\prime} \in V
$$

where $\mathrm{V}$ is the volume of a computational cell. The filter function, $G\left(x, x^{\prime}\right)$ implied here is then

$G\left(x, x^{\prime}\right)\left\{\begin{array}{c}1 / V, \quad x^{\prime} \in V \\ 0, \quad x^{\prime} \text { otherwise }\end{array}\right.$

Filtering the Navier-Stokes equations, one obtains for incompressible flow

$$
\frac{\partial \rho}{\partial t}+\frac{\partial}{\partial x_{i}}\left(\rho \bar{u}_{i}\right)=0
$$

and 


$$
\frac{\partial}{\partial t}\left(\rho \bar{u}_{i}\right)+\frac{\partial}{\partial x_{i}}\left(\rho \bar{u}_{i} \bar{u}_{j}\right)=\frac{\partial}{\partial x_{j}}\left(\mu \frac{\partial \sigma_{i j}}{\partial x_{j}}\right)-\frac{\partial \bar{p}}{\partial x_{i}}-\frac{\partial \tau_{i j}}{\partial x_{j}}
$$

where $\sigma_{i j}$ is the stress tensor due to molecular viscosity defined by

$$
\sigma_{i j} \equiv\left[\mu\left(\frac{\partial \bar{u}_{i}}{\partial x_{j}}+\frac{\partial \bar{u}_{j}}{\partial x_{i}}\right)\right]-\frac{2}{3} \mu \frac{\partial \bar{u}_{l}}{\partial x_{l}} \delta_{i j}
$$

and $\tau_{i j}$ is the subgrid-scale stress defined by

$$
\tau_{i j} \equiv \rho \overline{u_{i} u_{j}}-\rho \bar{u}_{i} \bar{u}_{j}
$$

\section{Subgrid-Scale Models}

The subgrid-scale stresses resulting from the filtering operation are unknown, and require modeling. The subgrid-scale turbulence models in FLUENT employ the Boussinesq hypothesis as in the RANS models, computing subgrid-scale turbulent stresses from

$$
\tau_{i j}-\frac{1}{3} \tau_{k k} \delta_{i j}=-2 \mu_{t} \bar{S}_{i j}
$$

Where $\mu_{t}$ is the subgrid-scale turbulent viscosity. The isotropic part of the subgrid-scale stresses $\tau_{k k}$ is not modeled, but added to the filtered static pressure term. $\bar{S}_{i j}$ is the rate-ofstrain tensor for the resolved scale defined by

$$
\bar{S}_{i j} \equiv \frac{1}{2}\left(\frac{\partial \bar{u}_{i}}{\partial x_{j}}+\frac{\partial \bar{u}_{j}}{\partial x_{i}}\right)
$$

FLUENT offers four models for $\mu_{t}$ : a modification of the Smagorinsky model (called Smagorinsky-Lilly by FLUENT) both in standard and dynamic form, the WALE model, and the dynamic kinetic energy subgrid-scale model (the four models are used in this study with no noticeable difference of the flow field).

\section{Smagorinsky Model}

This simple model was first proposed by Smagorinsky [15]. In the Smagorinsky model [15], the eddy-viscosity is modeled by

$\mu_{t}=\rho L_{s}^{2}|\bar{S}|$

Where $L_{s}$ is a length scale based on the filter width and $|\bar{S}| \equiv \sqrt{2 \bar{S}_{i j} \bar{S}_{i j}}$

In FLUENT, $L_{s}$ is computed using

$$
L_{s}=\min \left(\kappa d, C_{s} V^{1 / 3}\right)
$$

Where $\kappa$ is the von Kármán constant, $d$ is the distance to the closest wall, $C_{s}$ is the Smagorinsky constant, and $V$ is the volume of the computational cell. This model is referred 
to by FLUENT as Smagorinsky-Lilly. Close to the wall this model becomes a classical algebraic turbulence model with a Prandlt mixing length formulation.

Lilly derived a value of 0.17 for $C_{s}$ for homogeneous isotropic turbulence in the inertial subrange. However, this value was found to cause excessive damping of large-scale fluctuations in the presence of mean shear and in transitional flows as near solid boundary, and has to be reduced in such regions. In short, $C_{s}$ is not a universal constant, which is the most serious shortcoming of this simple model. Nonetheless, $C_{s}$ value of around 0.1 has been found to yield the best results for a wide range of flows, and is the default value in FLUENT.

\section{Dynamic Smagorinsky Model}

Germano et al. [16] and subsequently Lilly [17] conceived a procedure in which the Smagorinsky model constant, $C_{s}$ is dynamically computed based on the information provided by the resolved scales of motion. The dynamic procedure thus obviates the need for users to specify the model constant $C_{s}$ in advance.

The $C_{s}$ obtained using the dynamic Smagorinsky model varies in time and space over a fairly wide range. To avoid numerical instability, in FLUENT, $C_{s}$ is clipped at zero and 0.23 by default.

Other models available in FLUENT are the Wall-Adapting Local Eddy-Viscosity (WALE) model and the Dynamic Kinetic Energy Subgrid-Scale Model in which an additional equation is solved for the subgrid-scale kinetic energy. We refer to the literature, [18-19] and the FLUENT manual for details of these models.

\subsection{Solver Settings}

The finite volume method has been used to discretise the partial differential equations of the model using the SIMPLEC (Semi-Implicit Method for Pressure-Linked Equations-Consistent) method for pressure - velocity coupling and both the second order upwind scheme and QUICK scheme to interpolate the variables on the surface of the control volume (with no noticeable difference in results for using both of these schemes), For LES simulation stage, the bounded central difference scheme is the default convection scheme. The implicit coupled solution algorithm was selected. The Reynolds stress (RSM) turbulence model was used in this study at the beginning stage of the simulation and switching to LES (with time step equals $0.0001 \mathrm{~s}$ ) in a later stage. The inlet velocity is $13.5 \mathrm{~m} / \mathrm{s}$ for this study

The numerical grids of cyclones I, II and III contain 632000, 513000 and 413000 hexahedral cells respectively, to yield a reasonable prediction. These runs were performed on a 8 node Opteron Linux cluster. Run times were nearly $144 \mathrm{hrs}$ to complete $10^{4}$ time steps for LES (without particle injection study), for each case.

\section{Results and Discussion}

To understand the effect of changing cone-tip diameter on the flow field of cyclone separator, the flow field contours at the central section $(Y=0)$ are presented in this study, for more details about the flow field, line plots at six stations are extracted from the central section, viz. $z / D=0.5$ very close to the cone tip, $z / D=1.0, z / D=1.72, z / D=2.0, z / D=2.25$ passing through the inlet zone, and $z / D=2.66$ in the vortex finder zone are analyzed also. 
Table 2: The instantaneous contour plots for the three cyclones using LES (using dynamic kinetic energy subgrid-scale model), $t=2 \mathrm{~s}$

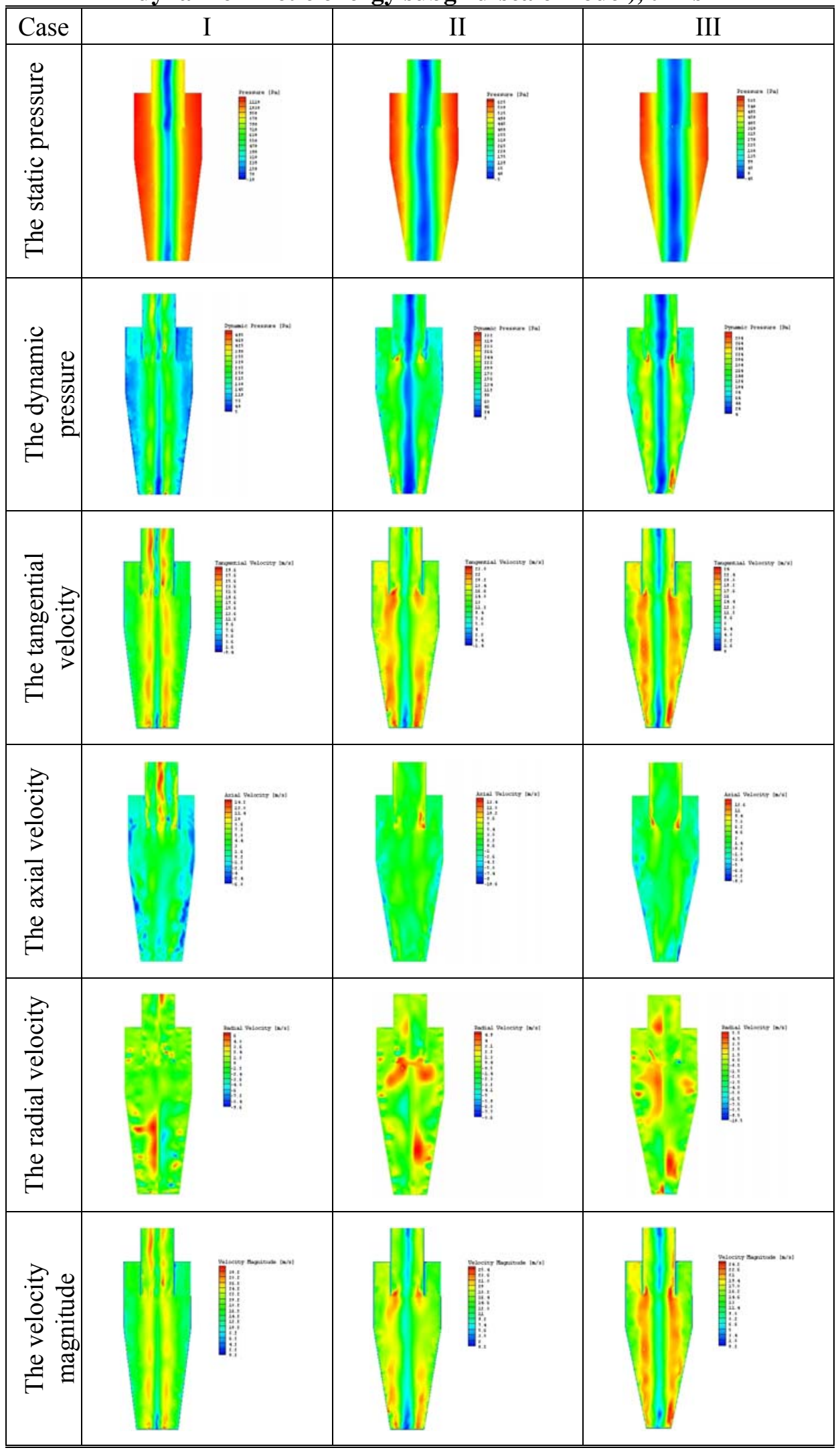


Table 3: The time-averaged contour plots for the three cyclones using LES (using dynamic kinetic energy subgrid-scale model

\begin{tabular}{|c|c|c|c|}
\hline Case & I & II & III \\
\hline 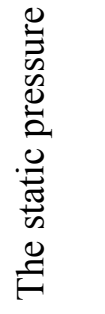 & 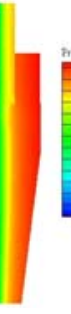 & & \\
\hline 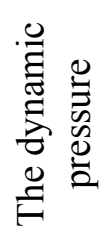 & 标 & 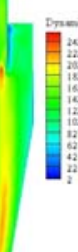 & \\
\hline 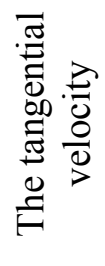 & 目: & 模 & \\
\hline 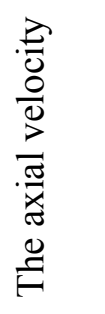 & 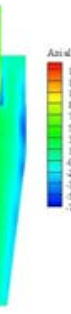 & 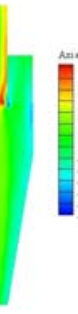 & \\
\hline 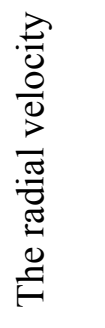 & 标 & 标 & \\
\hline 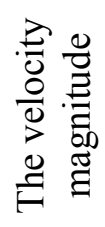 & 到 & 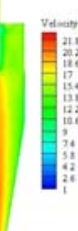 & E \\
\hline
\end{tabular}




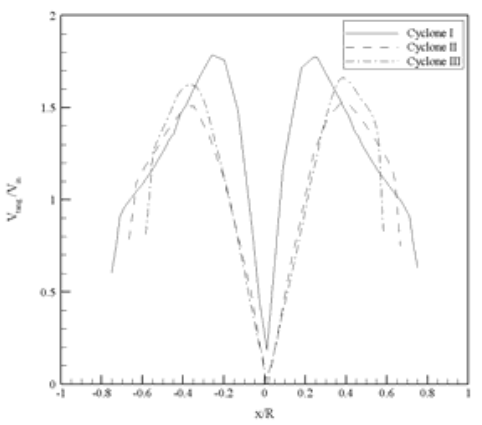

(a) At $z / D=0.5$

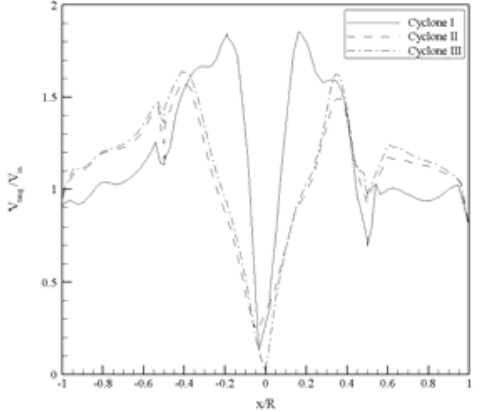

(d) At $z / D=2.0$

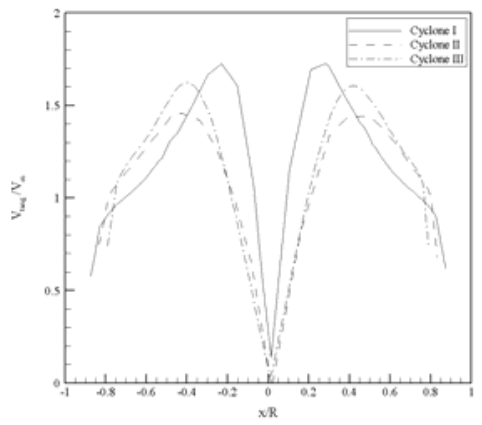

(b) At $z / D=1.0$

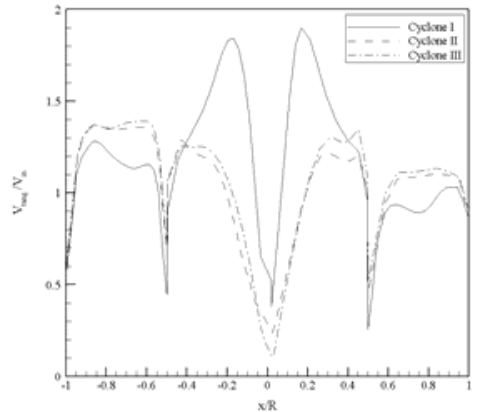

(e) At $z / D=2.25$

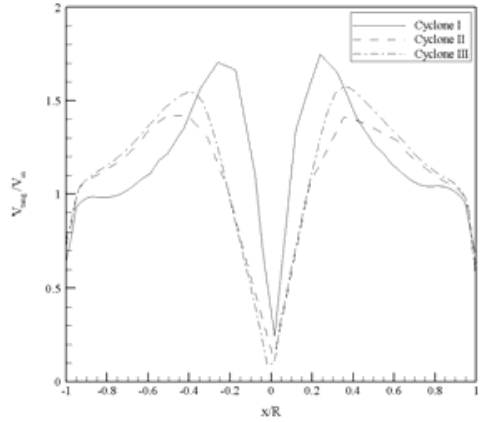

(c) At $z / D=1.72$

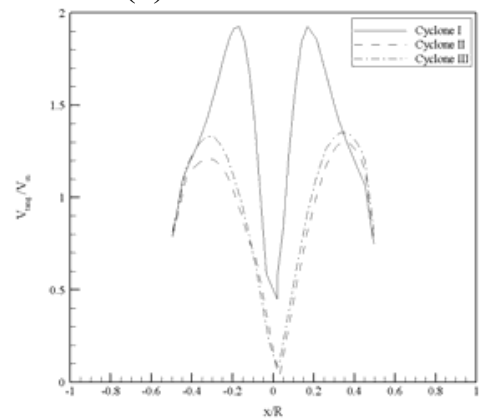

(f) At $z / D=2.66$

Fig. 2: The mean tangential velocity profiles for the three cyclones at five stations $(Y=0)$ (measured from the cyclone bottom).

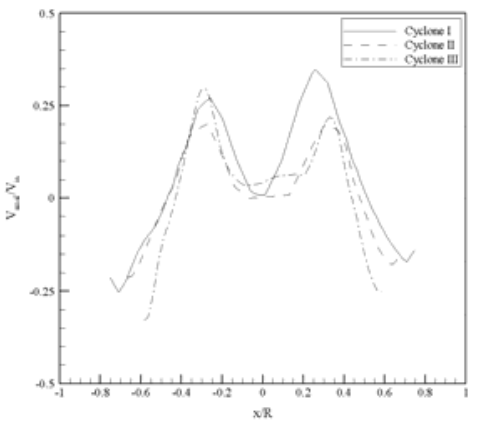

(a) At $z / D=0.5$

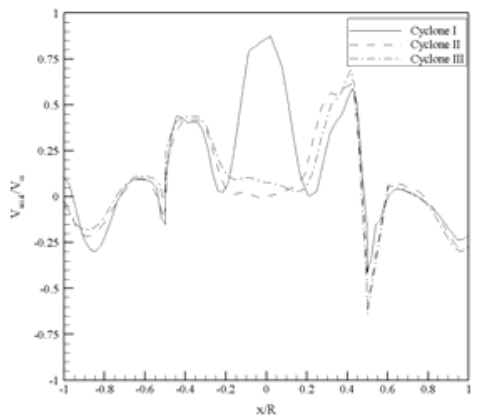

(d) At $z / D=2.0$

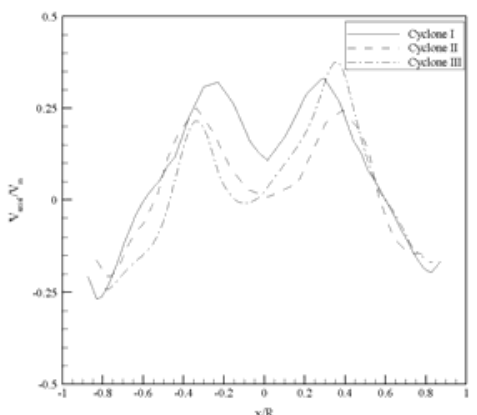

(b) At $z / D=1.0$

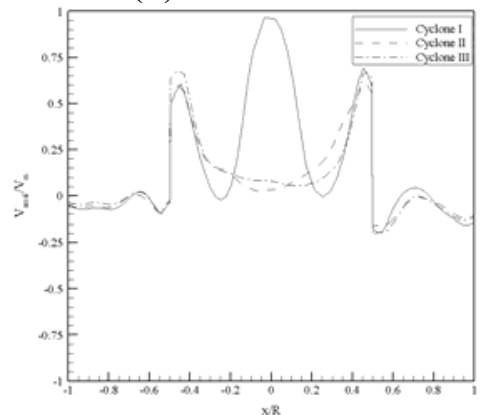

(e) At $z / D=2.25$

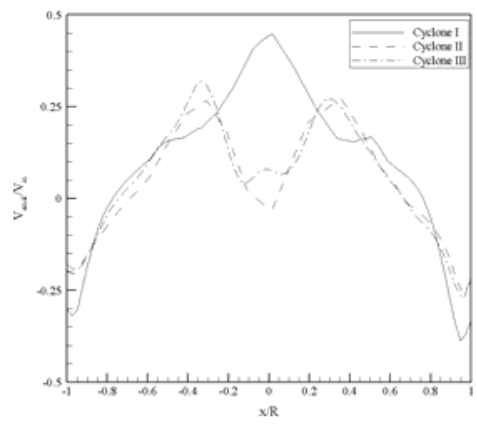

(c) At $z / D=1.72$

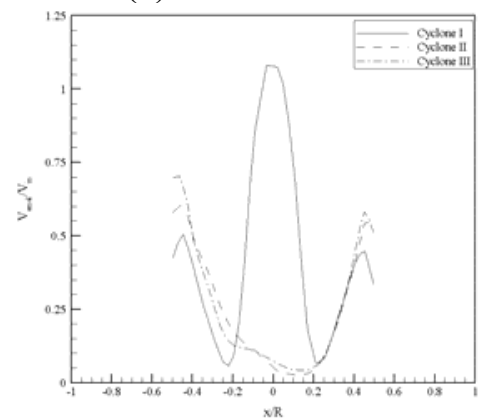

(f) At $z / D=2.66$

Fig. 3: The mean axial velocity profiles for the three cyclones at five stations $(Y=0)$ (measured from the cyclone bottom). 


\subsection{The Pressure Field}

The contour plots for static pressure for the three cyclones are presented in table 2 for LES (instantaneous value at $\mathrm{t}=2 \mathrm{~s}$ using dynamic kinetic energy subgrid-scale model) and table 3 for time averaged LES. The results show that, the static pressure decreases radially from wall to center, with a negative pressure zone appearing in the forced vortex region (central region due to high swirling velocity) allowing particles to escape if they enter this zone. Consequently, it is better to maintain the minimal pressure as large as possible which is the case for cyclone II which means that decreasing cone tip diameter increases the collection efficiency only if $B_{c} \geq D_{x}$, while if $B_{c}$ becomes less than $D_{x}$ the tangential velocity will increase considerably because the same amount of flow will occupy smaller volume, but also the minimum pressure value in the domain will decrease considerably and the chance of particles to enter this zone will increase and finally the centrifugal forces acting on the particles will fail to throw it to the wall.

The pressure drop in the cyclone, i.e. the difference between area average total pressure between inlet and outlet sections, is respectively $352,423,435 \mathrm{~Pa}$ for cyclones I, II, III (these values are higher than those of Xiang et al [5] but the same trend is found which indicates that, decreasing cone tip diameter increase pressure drop and also its effect on pressure drop is minor.)

The contour plot for static pressure also shows that the pressure gradient is the largest along radial direction (due to existing of forced vortex at the center) while the changes in axial direction are very small. The contour plot for dynamic pressure is the largest at the interface between forced vortex and quasi-free vortex zone, the distribution of dynamic pressure is asymmetrical due to the non-symmetry of the inlet velocity (one inlet). The changes of cone tip diameter $B_{c}$ affect the values and distribution as clear in the center of the cyclone and at the entrance of the vortex finder.

\subsection{The Tangential Velocity}

The tangential velocity distribution is similar to the dynamic pressure distribution. This means that the tangential velocity is the dominant velocity component in the cyclone. For the same reason the velocity magnitude contours are almost identical with those of the tangential velocity. The value of the tangential velocity equals zero on the wall and at the center of the cyclone. The high speed gas enters the inlet (pure radial flow) and is accelerated upto $1.5 \sim 2.0$ times of the inlet velocity value, Fig. 2 . Then the velocity decreases as the gas spins down and reaches a minimum at the center. There is a chaotic flow just below the entrance of vortex finder, as the gas flow collides with the follow-up flow.

The gas velocity decreases sharply at the outside surface of the vortex finder which causes the well-known "short-circuiting flow" phenomenon at the entrance of the vortex finder, resulting in a higher pressure drop and a decrease of collection efficiency (the same phenomenon was observed in literature, e.g. [20]. Comparing the tangential velocity distribution given by table 2, table 3, and Fig. 2 the flow field is nearly identical especially cyclones II and III, leading to the following conclusion: "the effect of changing the cone-tip diameter on the flow field is negligible and consequently its effect on the cyclone performance is negligible". 


\subsection{The Axial Velocity}

Figure 3 shows the axial velocity profiles for the three cyclones at six different axial stations. The results show that, the axial velocity value can be subdivided into three categories, the first is directed downward near the wall, and the second is directed upward in the majority of the cyclone space, while the third is directed downwards at the center line near the cone tip, Fig. $3(\mathrm{a}-\mathrm{c})$. It is clear that the change of axial velocity in radial direction is larger than in axial direction, and the axial velocity reaches a peak value when the gas flows into the vortex finder.

The mean axial velocity profiles are very similar for the three cyclones except near the entrance of the vortex finder or in the vortex finder itself. Cyclones II and III behave nearly identical while cyclone I behaves somewhat different especially near and inside the vortex finder, see Fig. 3 (d-f)

\subsection{The Radial Velocity}

The contour plots of the radial velocities for the three cyclones show that the forced vortex, at the cyclones center, is a twisted cylinder and that its axis is a curve (the same phenomenon is reported by Wang et al. [14]. The flow enters the cyclone radially (negative-inward) after which the radial velocity becomes rapidly zero. Afterwards it becomes positive due to the centrifugal effect. Around the vortex finder the radial velocity near the lip of the vortex finder becomes negative again (directing to the center). The effect of decreasing cone tip diameter on radial velocity distribution is negligible as clear from the contour plots.

\section{Conclusions and Future Work}

The CFD code FLUENT 6.3 with the LES turbulence model, predicts well the flow field and performance of cyclones of different cone dimensions (the pressure drop is overestimated).

LES results agree with Xiang's experiment that a cyclone with a smaller cone tip diameter $B_{c}$ results in a higher pressure drop compared to a cyclone with a bigger cone diameter. This is due to a higher velocity magnitude and Reynolds number inside the cyclone with a smaller cone size compared to a cyclone of a bigger cone size, but the effect of this geometrical parameter on the flow field and pressure drop is negligible. Results obtained from the computational modeling have demonstrated that CFD is an effective method for modeling the effect of cyclone dimension on its performance.

Further extensions of this work include the numerical prediction of the cyclone collection performance. For each cyclone this may be done by (1) solving the gas flow field using LES, and (2) Lagrangian tracking of a number of particle size classes to obtain complete grade efficiency curves. By this way, the performance of each cyclone can be determined in terms of pressure drop and cut-off size. Also comparison with the results from mathematical models will help to select the best mathematical model for sampling cyclones

\section{References}

[1] Büttner, H., 1988. Size separation of particles from aerosol samples using impactors and cyclones. Particle and Particle Systems Characterization Vol. 2, pp. 87-93.

[2] Iozia, D. L., Leith, D., 1989. Effect of cyclone dimensions on gas flow pattern and collection efficiency. Aerosol Science and Technology Vol. 10, pp. 491-500. 
[3] Kim, J. C., Lee, K. W., 1990. Experimental study of particle collection by small cyclones. Aerosol Science and Technology Vol. 12, pp. 1003-1015.

[4] Zhu, Y., Kim, M. C., Lee, K. W., Park, Y. O., Kuhlman, M. R., 2000. Design and performance evaluation of a novel double cyclone. Aerosol Science and Technology Vol. 34, pp. 373-380.

[5] Xiang, R., Park, S. H., Lee, K. W., 2001. Effects of cone dimension on cyclone performance. Journal of Aerosol Science, Vol. 32, pp. 549-561.

[6] Bryant, H. S., Silverman, R., Zenz, F. A., 1983. How dust in gas affects cyclone pressure drop? Hydrocarbon Processing, Vol. 62, pp. 87-90.

[7] Zhu, Y., Lee, K. W., 1999. Experimental study on small cyclones operating at high flow rates. Journal of Aerosol Science, Vol.30, pp. 1303-1315.

[8] Chuah, T., Gimbun, J., Choong, T. S., 2006. A CFD study of the effect of cone dimensions on sampling aerocyclones performance and hydrodynamics. Powder Technology, Vol. 162, pp. $126-132$.

[9] Barth, W., 1956. Berechnung und auslegung von zyklonabscheidern aufgrund neuerer untersuchungen. Journal of Aerosol Science Vol. 8, pp. 1-9.

[10] Leith, D., Licht, W., 1972. The collection efficiency of cyclone type particle collectors: a new theoretical approach. A.I.Ch.E. Symposium Series 68, pp. 196-206.

[11] Iozia, D. L., Leith, D., 1990. The logistic function and cyclone fractional efficiency. Aerosol Science and Technology, Vol. 12, pp 598-606.

[12] Griffiths, W. D., Boysan, F., 1996. Computational fluid dynamics (CFD) and empirical modeling of the performance of a number of cyclone samplers. Journal of Aerosol Science Vol. 27, pp. 281-304.

[13] Slack, M., 2003. "QNET-CFD Application Challenge, Cyclonic Separator". Last accessed $21 \quad$ December 2008, http://forum.abok.ru/index.php?act=attach\&type=post\&id=2702.

[14] Wang, B., Xu, D., Chu, K., Yu, A., 2006. Numerical study of gas-solid flow in a cyclone separator, Applied Mathematical Modelling Vol. 30, pp. 1326-1342.

[15] Smagorinsky, J., 1963. General circulation experiments with the primitive equations. The Basic Experimental Monththy Weather Revision, Vol. 91, pp 99-164.

[16] Germano, M., Piomelli, U., Moin, P. and Cabot, W. H., 1991. A Dynamic SubgridScale Eddy Viscosity Model, Physics of Fluids A, Vol. 3, No. 7, pp. 1760-1765.

[17] Lilly, D. K., 1991. A Proposed Modification of the Germano Subgrid-Scale Closure Method, Physics of Fluids A, Vol. 4, No. 3, pp. 633-635.

[18] Nicoud, F., Ducros, F., 1999. Subgrid-scale stress modelling based on the square of the velocity gradient tensor. Flow, Turbulence, and Combustion, Vol. 62, No. 3, pp. 183200.

[19] Kim, W. W., Menon, S., 1997. Application of the localized dynamic subgrid-scale model to turbulent wall-bounded flows. Technical Report AIAA-97-0210, AIAA,35 ${ }^{\text {th }}$ Aerospace Sciences Meeting, Reno, NV.

[20] Xiang, R. B., Lee, K. W., 2005. Numerical study of flow field in cyclones of different heights. Chemical Engineering and Processing, Vol. 44, pp. 877-883. 